\title{
Anillo lnterior de Santiago: Santiago mejor
}
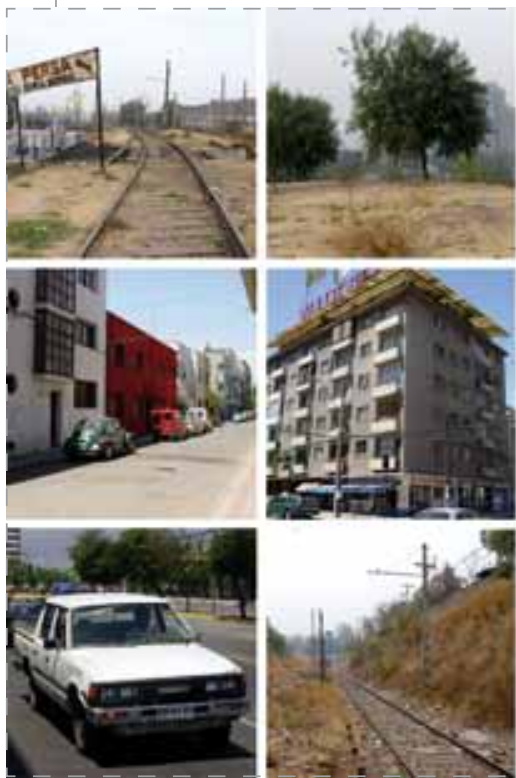
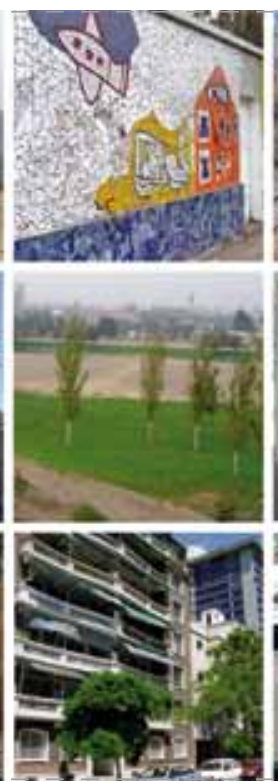
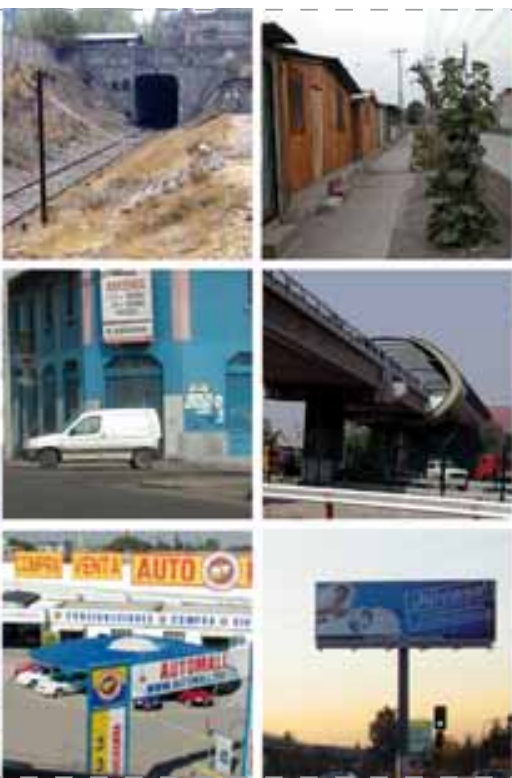
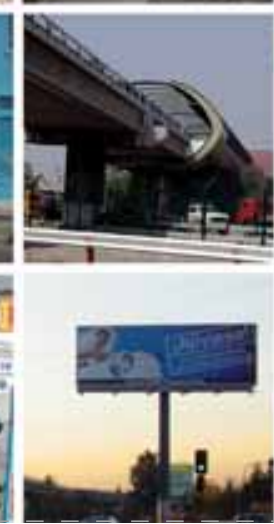
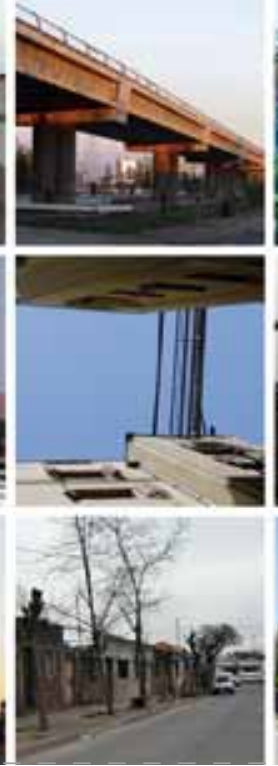

Ho Interior de Santiago, MINVU Diagramada por José Maldonado A.
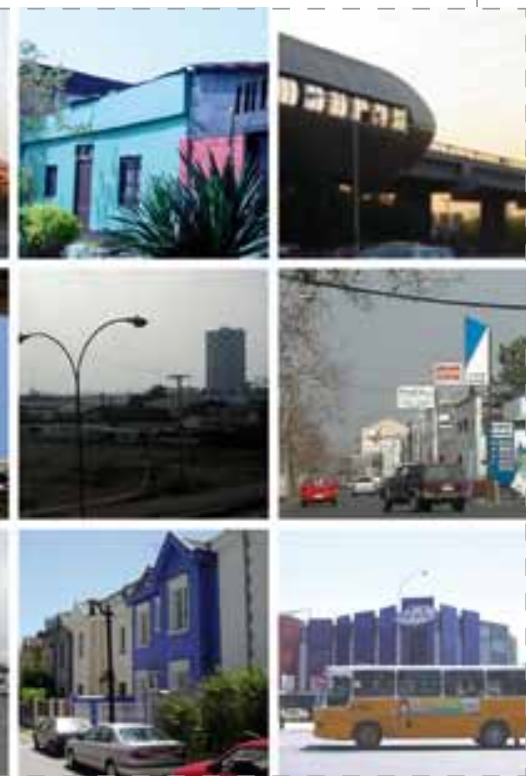

El proyecto Anillo Interior de Santiago es una Estrategia de Recuperación Urbana que está siendo impulsada por el Ministerio de Vivienda y Urbanismo de Chile como uno de los Proyectos Emblemáticos para la celebración del Bicentenario de la República el año 2010, y que tiene como objetivo hacer de Santiago una ciudad más eficiente, moderna y con mejor calidad de vida.

La estrategia apunta a mejorar las condiciones urbanas y ambientales de las cerca de 250 hectáreas de terrenos deteriorados y subutilizados que hoy se encuentran en el centro de la ciudad y que estaban relacionados al antiguo Ferrocarril de Cintura de Santiago, con el fin de generar un proceso de renovación urbana que permita cambiar la imagen y el estándar del centro de Santiago.

Las principales ventajas de la implementación de este proyecto son:

- Densifica el área central evitando el crecimiento en extensión de la ciudad.

- Aprovecha la infraestructura existente y evita nuevas inversiones del sector público en la periferia.

- Evita la segregación urbana al equipar un lugar de la ciudad accesible para todos los habitantes.

- Descontamina Santiago al disminuir los viajes de la población a sus lugares de trabajo.

- Fomenta el turismo, genera riqueza y nuevas fuentes de trabajo.

- Protege los barrios históricos, el patrimonio natural y edificado.

El área del Anillo Interior abarca un sector intercomunal central de la ciudad de Santiago que se estructura sobre cuatro ejes: dos sistemas de Parques (Parque Forestal y de los Reyes al norte y Parque La Aguada al sur) y dos bulevares (Av. Exposición y Matucana al poniente, Av. Vicuña Mackenna al oriente).

El trazado hoy coincide con el límite de la comuna de Santiago y las doce comunas que la circundan (Providencia, Recoleta, Independencia, Renca, Quinta Normal, Estación Central, Cerrillos, Pedro Aguirre Cerda, San Miguel, San Joaquín, Macul y Ñuñoa) abarcando una población de 1.542.298 habitantes según censo 2002.

El Plan Maestro para el área apunta a hacer del Anillo un sistema de espacios públicos y parques (Anillo Verde), que aproveche las inversiones en infraestructura de transporte (Anillo Conectado), que vincule elementos culturales y patrimoniales (Anillo Cultural), que integre los barrios heterogéneos del área (Anillo Diverso) y que fomente la renovación urbana (Anillo Renovado). 


\section{Santi ago I nner Ring: \\ A better city}

The Santiago Inner Ring project is an urban regeneration strategy which is being promoted by the Chilean Ministry for Housing and Urbanism as one of the emblematic projects of the Bicentennial celebration of Chile in 2010. Its objective is to make Santiago into a more efficient and modern city with a better quality of life for its inhabitants.

The strategy aims to improve the urban and environmental conditions of the approximately 250 hectares of derelict and underused land now located in the city centre and formerly related to Chile's old ring railway. The project seeks to generate a process of urban regeneration which will allow the image of and living standards in Santiago's city centre to improve.

The main advantages of implementing this project are as follows:

- The project will encourage densification in the city centre and therefore help slow down further urban sprawl on the periphery of Santiago.

- It takes advantage of existing infrastructure and thus avoids new public investments on the periphery.

- It avoids urban segregation by providing public and recreational space within the city that is accessible to all its inhabitants.

- The project helps decontaminate Santiago by reducing commuting times and distances for its inhabitants.

- It promotes tourism, generates wealth and provides new sources of work.

- The project protects historic town quarters, as well as the natural and architectural heritage of the city.

The area of the Santiago Inner Ring project encompasses an inter-municipal and central area of the city that is structured on four axes: two park systems (Parque Forestal and Parque de los Reyes to the north, and Parque La Aguada to the south) and two boulevards (Av. Exposición and Av. Matucana to the west and Av. Vicuña Mackenna to the east).

This route coincides with the limit of the municipality of Santiago with the twelve surrounding municipalities (Providencia, Recoleta, Independencia, Renca, Quinta Normal, Estación Central, Cerrillos, Pedro Aguirre Cerda, San Miguel, San Joaquin, Macul and Nuñoa) which together constitute a population of 1.542.298 inhabitants (2002 census).

The master plan for the area aims to transform the Santiago Inner Ring into a system of public space and parks (Green Ring) that takes advantage of various investments in transport infrastructure (Connected Ring), tying together cultural and patrimonial elements (Cultural Ring) and integrating heterogeneous city districts (Diverse Ring) to encourage urban regeneration (Regenerated Ring).

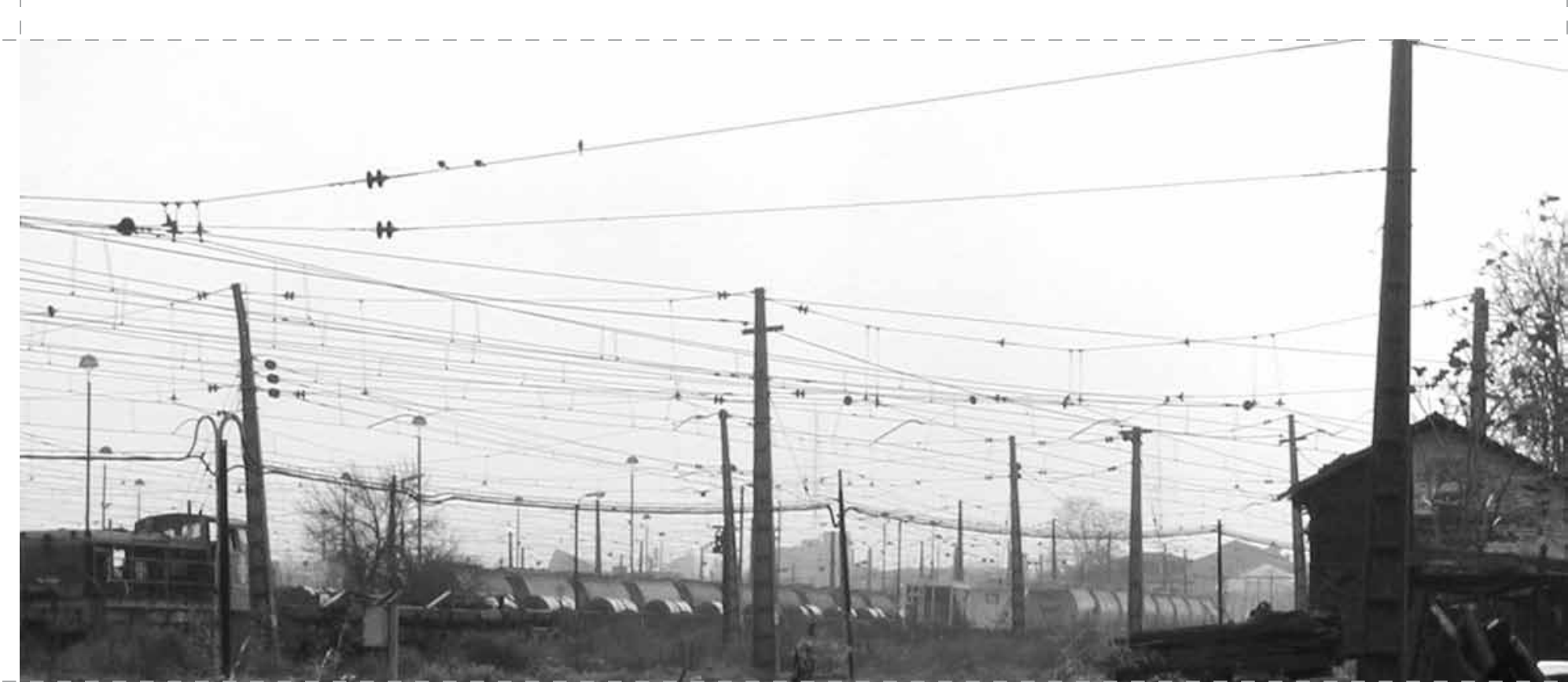




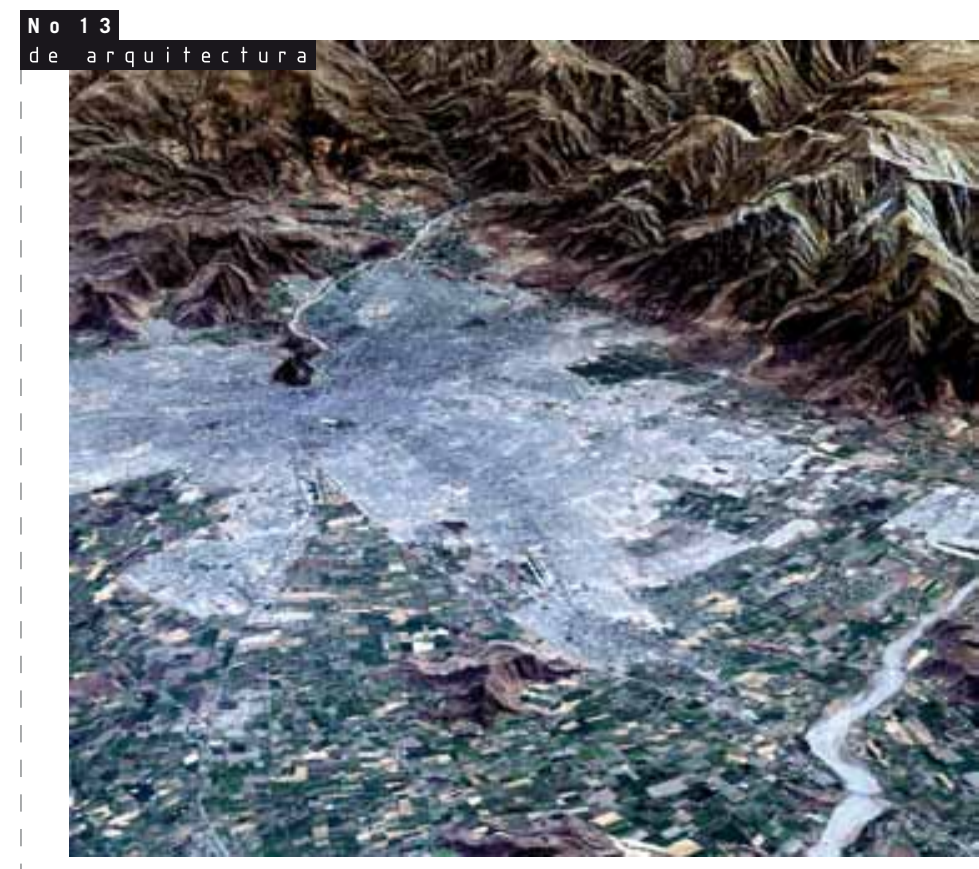

Cuenca de Santiago. Vista aérea.
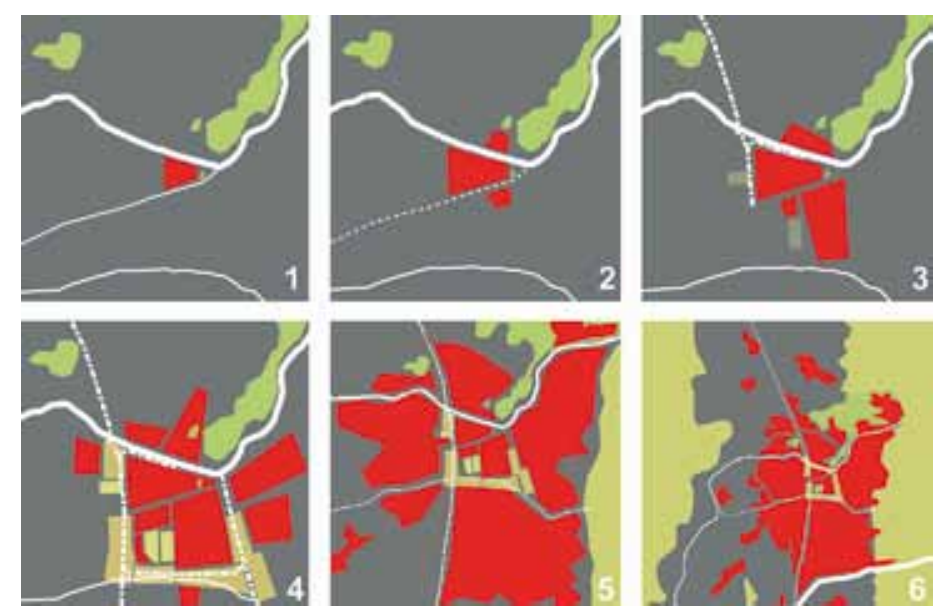

Etapas de crecimiento de Santiago: 1. Fundación, 2. Expansión limites naturales, 3. Contención limites antrópicos. 4.Expansión sobre los limites antrópicos, 5. Expansión hasta limites naturales y 6. Expansión por sobre los limites naturales y Regeneración de vacíos interiores (R. Moris, 2002).
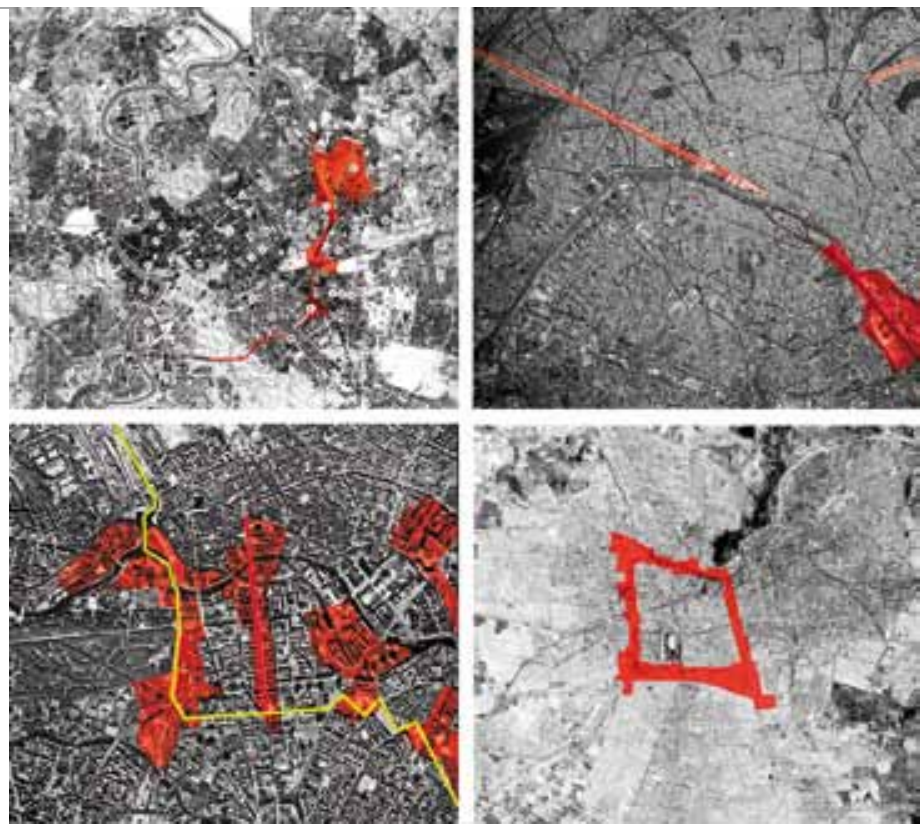

Áreas de renovación urbana: Roma / Paris / Berlín / Santiago.

Anillo interior de Santiago:

Origen del proyecto

La propuesta de desarrollar un gran proyecto para la ciudad de Santiago emerge desde dos ámbitos distintos, recuperando los terrenos de lo que fuera el antiguo cinturón ferroviario, que marco el limite de la ciudad hasta principios del siglo xx y que fue siendo sobrepasado sostenidamente desde entonces, sin que se perdiera la condición de periferia urbana de gran parte de su perímetro. Terrenos y usos centrales, deteriorados y obsolescentes, vinculados a este cinturón ferroviario son reconocidos como un desafío, a la vez que una gran oportunidad para reformular la organización de la ciudad de Santiago en el siglo xxI.

\section{Áreas de renovación}

El reconocimiento de este origen histórico y de los potenciales asociados a la suma de oportunidades y fortalezas del lugar, nos remite a las experiencias de tantas ciudades en el mundo que han estructurado sus planes de crecimiento y Renovación Urbana en torno de áreas obsoletas y físicamente deterioradas, aunque con gran potencial de centralidad. Madrid, Bilbao, París, Roma, Praga, Buenos Aires, sólo por citar algunos casos, han sido ciudades en las que se han llevado a cabo experiencias de gestión urbana que han logrado redefinir o reorientar el destino de áreas que sin una estrategia eficiente de administración permanecerían aún representando un obstáculo para el desarrollo de la ciudades.

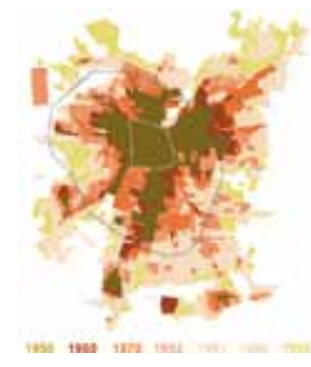

Crecimiento de mancha urbana de Santiago (1950-1996). Fuente: Atisba Consultores.

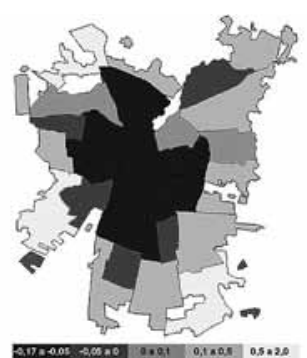

Tasas de despoblamiento. Fuente: censo 2002

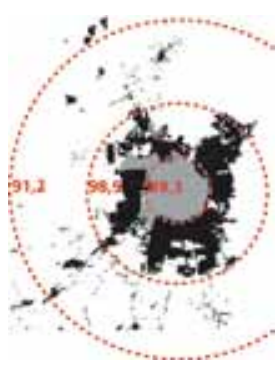

Densidades de población Santiago 2002. Fuente: Levantamiento Atisba Consultores. 


\section{Los nuevos desarrollos interiores}

La ciudad está creciendo en base a dos grandes procesos: el de expansión fuera del límite urbano y el de crecimiento de comunas periféricas. Dentro de esta transformación, la ciudad presenta un proceso de densificación sólo en las comunas de Santiago, San Miguel y la Cuña Oriente.

Los fenómenos de expansión de Maipú, Puente Alto, Quilicura y San Bernardo, se han desarrollado con proyectos orientados a los estratos C3 y D, mientras Maipú y La Florida ofrecen alternativas principalmente al estrato C2. Peñalolén y Huechuraba son alternativas accesibles para los estratos C1 y $\mathrm{C} 2$.

La Intercomuna Central concentró el 2do. semestre del 2001 el 50,9\% de la oferta y el 55,8\% de la venta de departamentos, con oferta en nueve de las trece comunas y con sólo Santiago, Providencia, Ñuñoa, Macul y San Miguel presentando cifras relevantes. De este total, las comunas vinculadas socio-económicamente al área oriente (Providencia y Ñuñoa) representan el 22,5\% de la venta del área. Por otro lado, el sector oriente, reunió a las comunas de Las Condes, Vitacura y Lo Barnechea el 39,0\% de la oferta y el $32,2 \%$ de la venta de departamentos.

El fenómeno de consolidación de la Cuña Oriente y su descolgamiento del distrito central (CBD), es comparable a la espina de desarrollo que detectan, E. Griffin y L. Ford, (1980), en las ciudades latinoamericanas, esto se puede observar en la aplicación del modelo en Santiago donde las zonas de repulsión están representadas por la localización de industrias y usos deteriorantes.
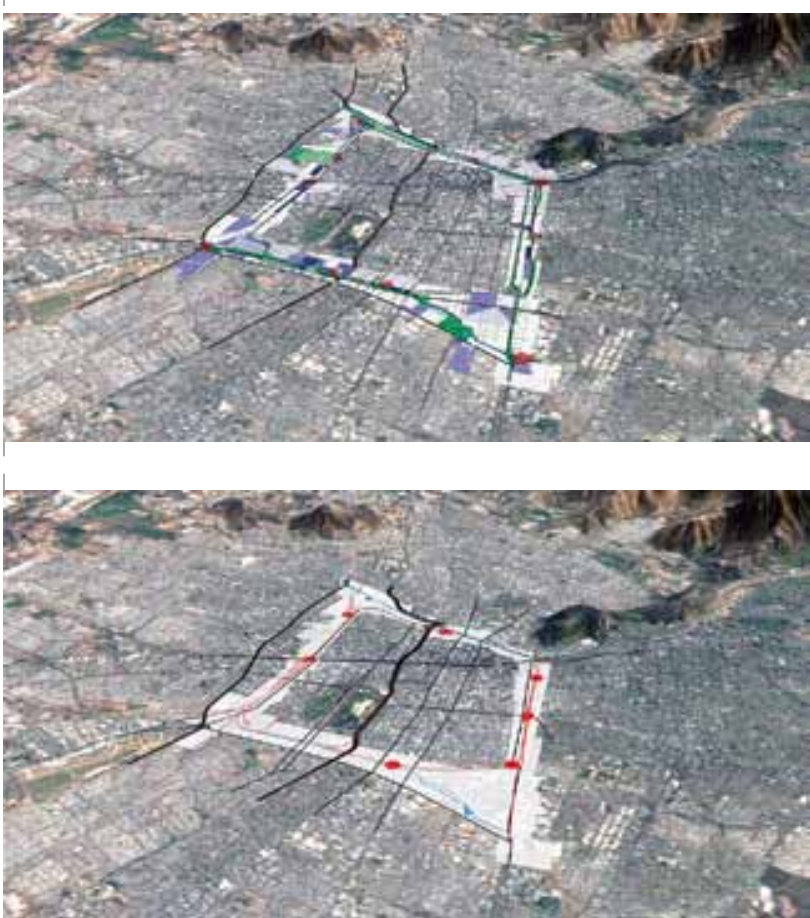
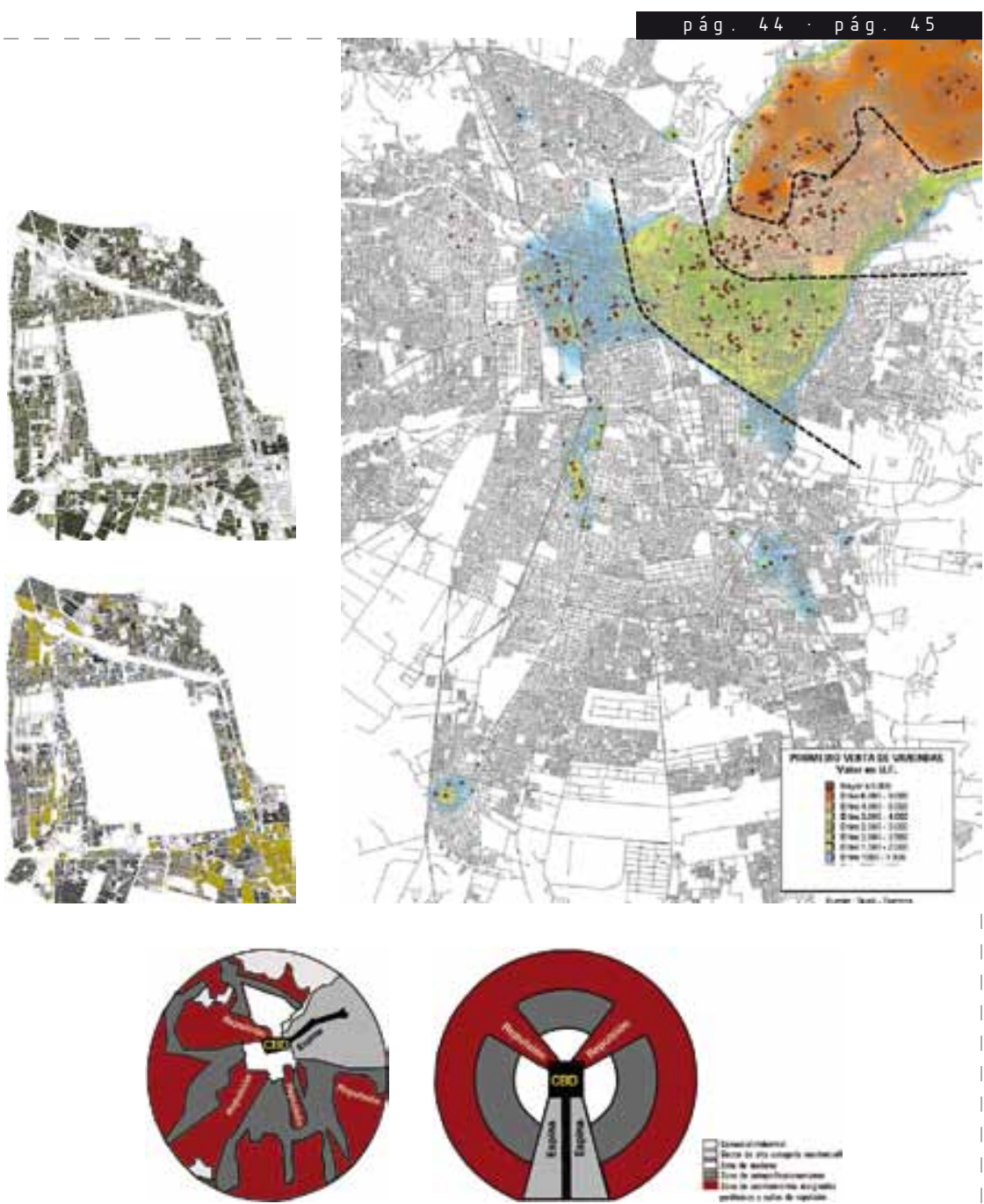

Modelo de crecimiento de Griffin \& Ford, aplicado a la ciudad de Santiago. Fuente: Tesis "Frontera Interior de Santiago" (Moris \& Reyes, 1999).

\section{Di agnósti co estratégi co territorial}

- El anillo es un área urbana heterogénea y multifuncional. Presenta una diversidad de usos de suelo, actividades urbanas, grados de consolidación, calidad urbana estados de espacios públicos y áreas verdes, etcétera.

- La normativa urbana no reconoce el Anillo Interior. La normativa no reconoce el Anillo en ninguna de sus escalas instrumentales. Es decir, ni en el PRMS1, ni en los Planes Reguladores, ni en los Planes Seccionales vigentes en el área.

- En aspectos socio-demográficos, el Anillo presenta la heterogeneidad social y potencial de repoblamiento, aunque se observa cierta tendencia al despoblamiento del área. El anillo incluye un amplio abanico de estratos.

- La dinámica inmobiliaria del Anillo se concentra en focos o ejes de actividad, concentrada en productos multifamiliares de 900 a $22.00 \mathrm{UF}$ (27 a $32 \mathrm{UF} / \mathrm{m}^{2}$ ).

- El anillo posee un rol relevante -a nivel metropolitano- en cuanto al volumen de actividad económica y productiva.

- Las condiciones y dotaciones de infraestructuras de servicios se observan óptimas y no constituyen restricciones para el potencial desarrollo o renovación del área Anillo.

- El Anillo posee en la actualidad una buena conectividad y accesibilidad de escala macro (nacional, regional, metropolitana), tendiendo a optimizarse conforme a los proyectos de transporte previstos.

- El anillo presenta una importante oferta de suelo para desarrollo y/o renovación. 


\section{Anillo Verde / Gr een Ring}

La conformación de un espacio público continuo a lo largo del Anillo Interior constituye un componente clave de la propuesta para mejorar la imagen ambiental del centro de Santiago

Esto permitirá recorrer e integrar diferentes barrios de la ciudad, recuperar la escala humana y contribuir a un medio ambiente más sano, limpio y seguro.

Se propone crear un circuito de espacios públicos, parques, ciclovías, deporte y cultura, conformado por el Parque Forestal y de los Reyes al norte, las Avenidas Matucana y Exposición al poniente, El Parque la Aguada al sur y la Av. Vicuña Mackenna al oriente.

\section{Anillo Conectado \\ Connected Ring}

El Anillo Interior posee una excelente conectividad y accesibilidad a nivel metropolitano, nacional e internacional debido a la cercanía con el aeropuerto, la existencia de grandes autopistas, líneas de Metro, ejes del transporte público, terminales de buses interurbanos, y la presencia en el área de la Estación Central de Ferrocarriles.

Por ello, el proyecto propone aprovechar la confluencia de los diversos medios de transporte para potenciar el área como un territorio de gran centralidad único para la instalación de nuevas actividades vinculadas con el Gran Santiago y Chile.

\section{Anillo Cultural / Cul tural Ring}

En su recorrido, el Anillo Interior ofrece una oferta cultural a los habitantes de Santiago que permite reconocer barrios y obras arquitectónicas de valor patrimonial, destacando aquellas que fueron realizadas durante el Centenario de la República o durante el auge industrial y ferroviario de Santiago.

La propuesta del Anillo Interior contempla la integración, recuperación y puesta en valor de dichas obras, como una forma de reforzar la identidad de la ciudad permitiendo generar un sentido de pertenencia con sus habitantes y mostrando una nueva imagen de Santiago al exterior.

\section{Area Anillo Interice \\ - Rio Mapochio, Zanjón de la Aguada \\ Areas verdes existentes \\ 1-Áseas verdes existentes dentro del Anillo \\ Áreas verdes propuestas}

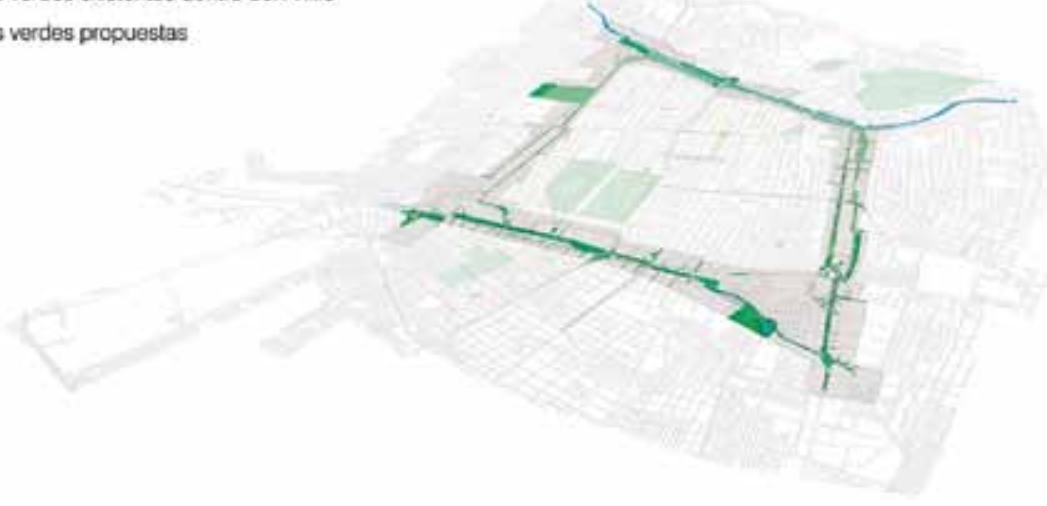

- Área Anillo Interior

\section{- Rio Mapocho, Zanjón de la Aguada \\ - Metro \\ ..... Mejoramiento de perfil vial \\ — Nuevas vialidades \\ - Autopistas concecionadas \\ — Vialidades existentes}

Area Anilo interior
- Rio Mapocho, Zanjón de la Aguada
Ereas verdes existentes
E Educacion
- Culto
E Patrimonio industrial
- Lugares de patrimonio y cultura
Estadios
E Comercio
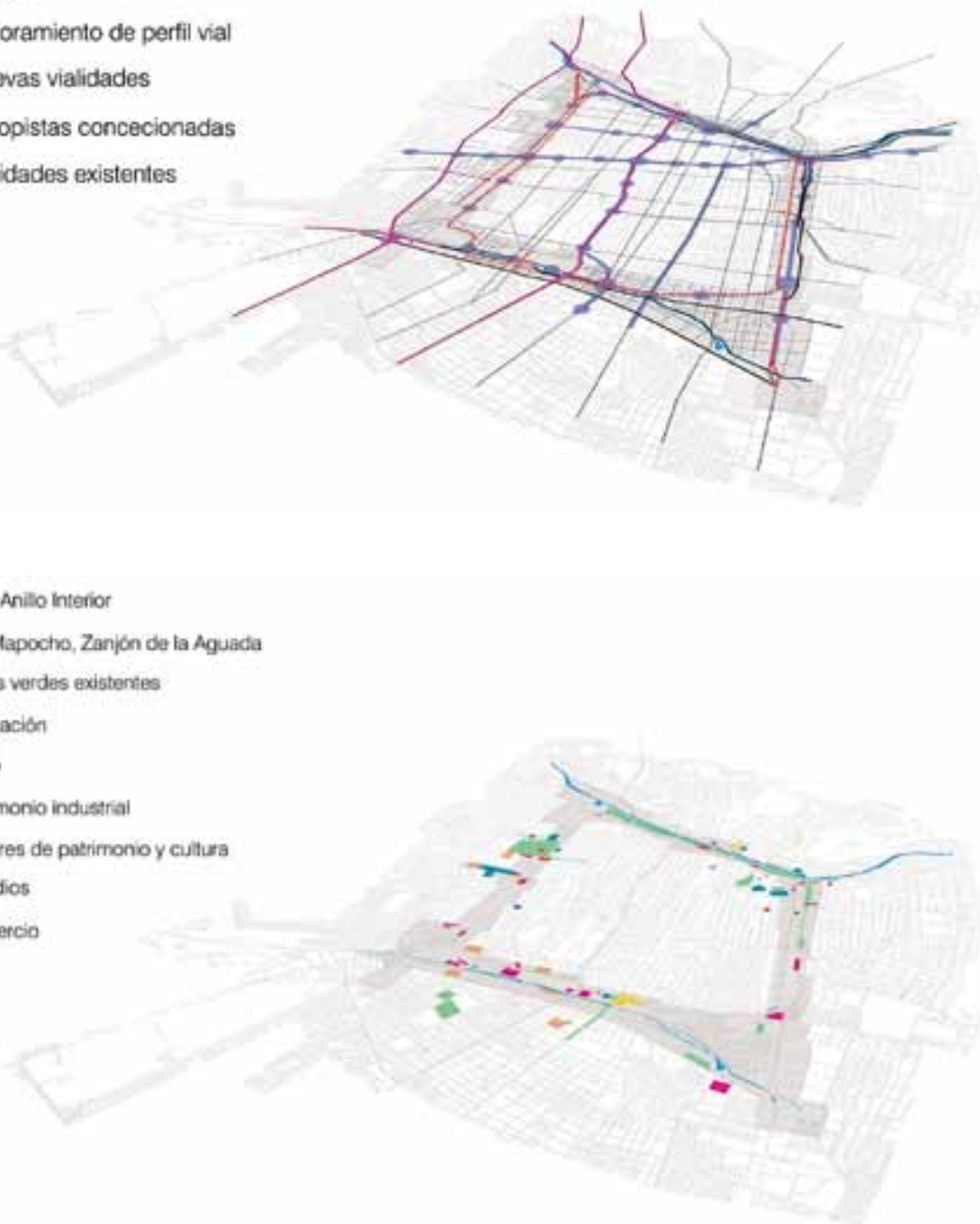


\section{Anillo Renovado / Renovated Ring}

El área del Anillo presenta una importante oferta de suelo para desarrollo y/o renovación urbana con óptimas condiciones y dotaciones de infraestructura de servicios. En conjunto los terrenos con potencial de desarrollo suman unas 250 hectáreas que corresponden a sitios eriazos, grandes paños, y terrenos con edificación deteriorada, que en gran parte son propiedad de Ferrocarriles, Bienes Nacionales, del SERVIU y de algunos Municipios. La propuesta entonces, apunta al desarrollo de dichos terrenos, permitiendo la intensificación del uso del suelo con actividades residenciales, de equipamiento, servicios y productivas, que permitan revitalizar el área y mejoren el estándar del centro de Santiago.

\section{Anillo Diverso/}

Di verse Ring

El área del Anillo Interior, es hoy un territorio de gran vitalidad por la presencia de diversos barrios y actividades como comercio, servicios, cultura, educación, vivienda y equipamiento.

Estas áreas muchas veces coexisten de manera desordenada y desagregada, generando fricción y deterioro urbano.

Es por ello, que se propone ordenar, promover y reforzar la condición heterogénea y multifuncional de actividades y habitantes, que presenta el Anillo Interior, para hacer de esta área un territorio urbano diverso, accesible para toda la ciudadanía y grato para vivir.

\section{Proyectos Prioritarios / Priority Projects}

El plan de desarrollo urbano total definido en el Plan Maestro para el Anillo Interior, tiene un horizonte de concreción de 30 años. La implementación de la primera fase está contemplada para el Bicentenario de la República el año 2010 e implica la construcción de los siguientes proyectos detonantes de desarrollo urbano:

- Parque la Aguada.

- Nuevo Centro Yungay y IV etapa del Parque de los Reyes.

- Reutilización Antigua Faja Vía.

- Reconversión urbana Maestranza San Eugenio.

\section{Área Anillo Interior}
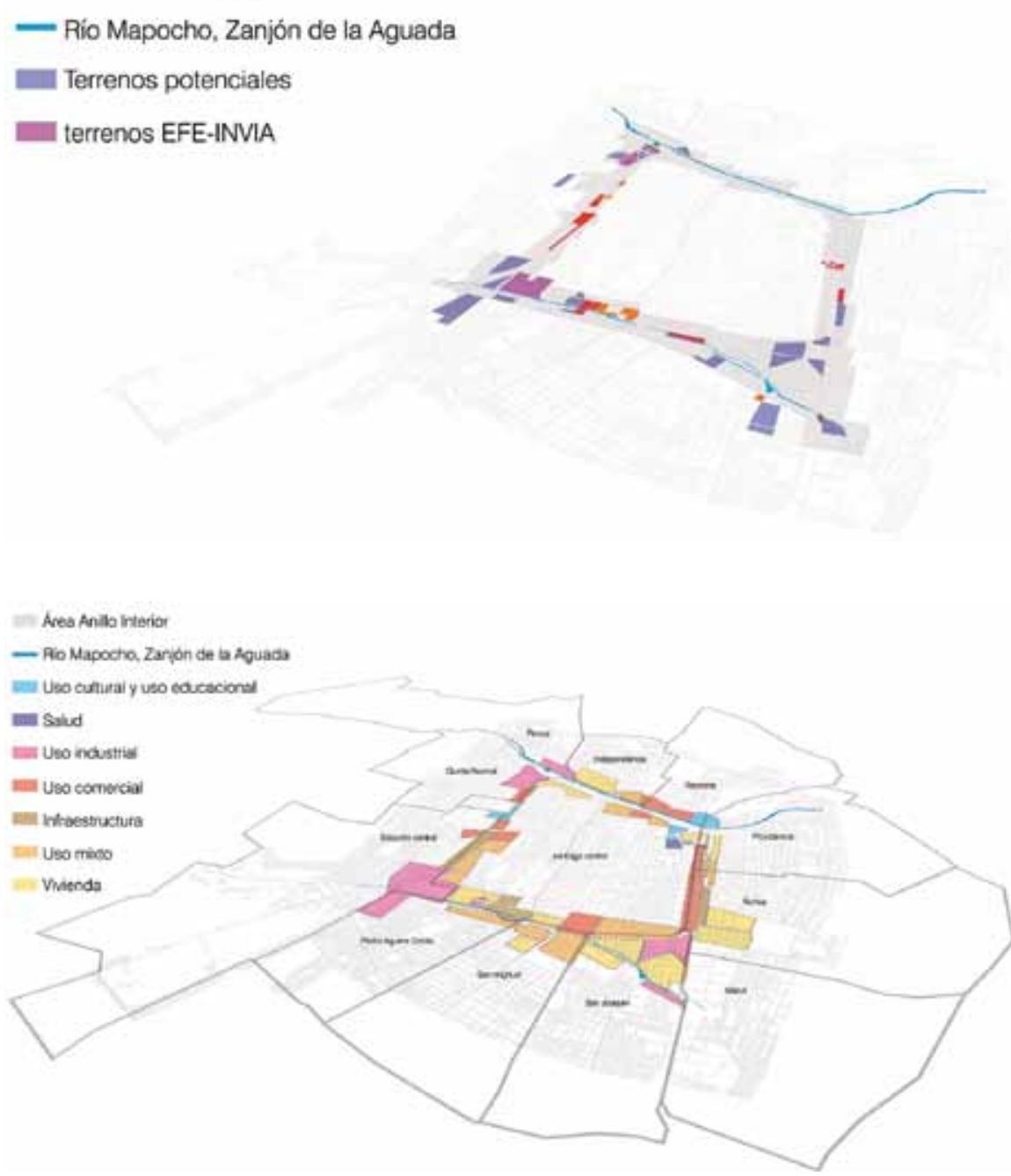

\section{Área Anilio Interior}

- Rilo Mapocho, Zanjón de la Aguada

- Anillo verde

Terrenos de recuperación urbana

.... Recuperación de la antigua faja vía

- Proyectos detonantes

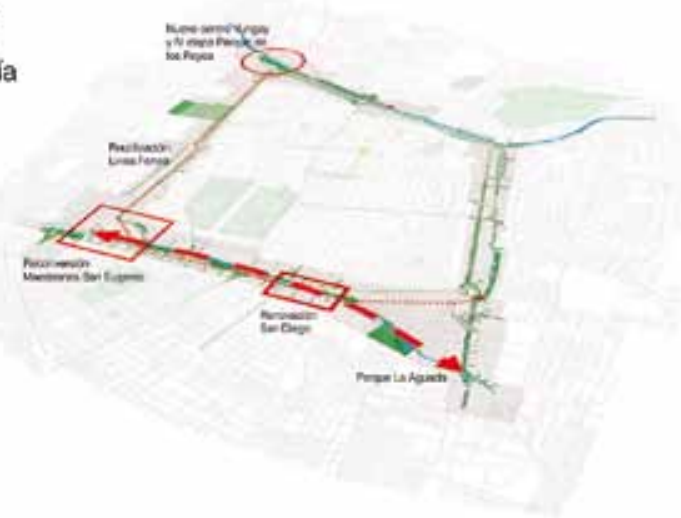

